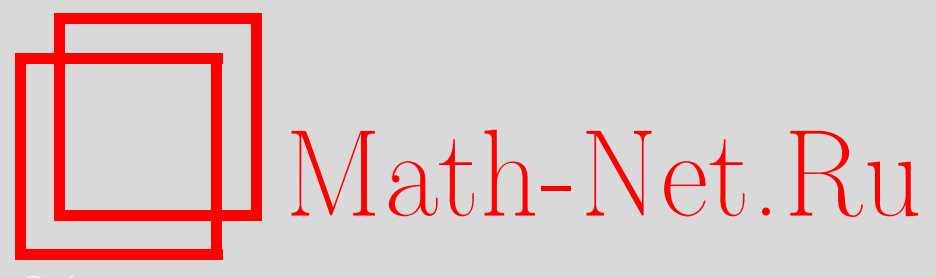

Д. В. Матюхин, В. А. Шишкин, Некоторые методы анализа функций хэширования и их применение к алгоритму ГОСТ Р 34.11-94, Матем. вопр. криптогр., 2012, том 3, выпуск 4, 7189

DOI: https://doi.org/10.4213/mvk68

Использование Общероссийского математического портала Math-Net.Ru подразумевает, что вы прочитали и согласны с пользовательским соглашением http: //www.mathnet.ru/rus/agreement

Параметры загрузки:

IP : 54.172 .240 .79

26 апреля 2023 г., 16:40:42 
УДК: 519.719.2+519.248:[004+007+654]

\title{
Некоторые методы анализа функций хэширования и их применение к алгоритму ГОСТ Р 34.11-94
}

\author{
Д. В. Матюхин, В.А. Шишкин
}

Технический комитет по стандартизации (ТК 26), Москва

Получено 20.V.2011

Исследуются криптографические функции хэширования, использующие завершающее преобразование специального вида. Для функций из этого класса (содержащего хэш-функцию ГОСТ Р 34.11-94) предложен новый метод построения мультиколлизии; показано, что метод Вагнера позволяет конструктивно описать большое число различных коллизий. Для хэш-функции ГОСТ Р 34.11-94 предложена модификация известного метода построения коллизии, использующая меньший объем памяти. Получена нижняя оценка объема используемой памяти, при котором трудоемкость модифицированного метода меньше трудоемкости универсальных методов построения коллизии.

Ключевые слова: функция хэширования, коллизия, мультиколлизия, ГОСТ Р 34.11-94, метод Вагнера

Some methods of hash functions analysis with application to the GOST P 34.11-94 algorithm

\section{V. Matyukhin, V. A. Shishkin \\ Russian Technical Committee for Standardization (TC 26), Moscow}

Abstract. A class of hash functions with a special finalization round (containing the GOST R 34.11-94 hash function) is considered. For functions from this class we propose a new multicollision attack and show that by means of Wagner's method it is possible to construct a description of a large number of different collisions. For GOST R 34.1194 hash function we propose a modification of the known collision attack using smaller amount of memory. We obtain lower estimate for the amount of memory permitting the modified attack to be faster than generic ones.

Key words: hash function, collision, multicollision, GOST R 34.11-94, Wagner's method Citation: Mathematical Aspects of Cryptography, 2012, vol. 3, no. 4, pp. 71-89 (Russian). 
В 2008 году австрийскими и польскими учеными $[14,15]$ для хэшфункции ГОСТ Р 34.11-94 были предложены алгоритмы построения прообраза и коллизии с трудоемкостью меньше «универсальных» оценок $2^{256}$ и $2^{128}$ соответственно. Отдельными этапами этих алгоритмов являются применение метода Вагнера [19] и одного метода построения мультиколлизии.

В настоящей статье рассматриваются существующие и предложен новый метод построения мультиколлизий функций хэширования. Оценивается эффективность применения этих методов к функциям хэширования, в которых функция сжатия применяется к контрольной сумме всех блоков сообщения, в частности, к хэш-функции ГОСТ Р 34.11-94.

Уточнена схема применения метода Вагнера в предложенном на CRYPTO'08 [15] алгоритме построения коллизии, получена оценка объема памяти. Предложена модификация этого алгоритма в условиях ограниченной памяти и получена оценка минимального объема памяти, при котором трудоемкость модифицированного алгоритма меньше, чем при использовании парадокса дней рождения.

\section{1. Криптографические функции хэширования}

Функиией хэширования называется отображение $H: V^{*} \rightarrow V_{n}$, где $n \in \mathbb{N}-$ натуральное число, $V^{*}-$ множество всех двоичных векторов (строк) конечной размерности (включая пустую строку), $V_{n}-$ множество всех $n$-мерных двоичных векторов.

Мы будем рассматривать только криптографические функиии хэширования, т. е. те функции хэширования, которые должны удовлетворять криптографическим требованиям. Более того, мы используем термины функция хэширования и хэщ-функиия для обозначения именно криптографических функций хэширования.

Аргумент $M \in V^{*}$ функции хэширования называется сообщением. Частью сообщения называется любой подвектор сообщения $M$. Хэщ-кодом (хэм-значением) сообщения $M \in V^{*}$ называется значение $H(M) \in V_{n}$.

На сегодняшний день наиболее распространены функции хэширования, построенные по итеративному принципу (итеративные функции хэмирования). Он восходит к работам $[6,16]$ Р. Меркля (Ralph C. Merkle) и И. Дамгорда (Ivan Damgård) 1989 года, в связи с чем итеративная конструкция получила название конструкции Меркля-Дамгорда. Конструкция Меркля-Дамгорда позволяет свести задачу построения хэш-функции $H: V^{*} \rightarrow V_{n}$ к задаче построения отображения $g: V_{q} \times V_{l} \rightarrow V_{q}$ с определенными свойствами. По итеративному принципу построены такие хэш-функции, как MD5 [17], SHA-1 
[3, 4], семейство хэш-функций SHA-2 [4], хэш-функции ГОСТ P 34.11-94 и ГОСТ Р 34.11-2012.

Опишем итеративные хэш-функции в общем виде и введем обозначения, которые будут использоваться в дальнейшем.

Перед вычислением значения итеративной функции хэширования исходное сообщение $M \in V^{*}$ преобразуется в сообщение $\bar{M} \in V^{*}$, длина которого кратна $l$. Данное преобразование называется процедурой дополнения сообщения (message padding), а сообщение $\bar{M}$ - расширенным сообщением (padded message). Дополнение сообщения может осуществляться различными способами, но, как правило, включает два этапа: конкатенация сообщения со значением его длины, записанным в двоичном виде, и с некоторой последовательностью до длины, кратной $l$, так, чтобы каждому исходному сообщению соответствовало единственное расширенное сообщение.

Пусть теперь $\bar{M}$ - сообщение, полученное с помощью процедуры дополнения сообщения $M$, и $\bar{M}=m_{1}\left\|m_{2}\right\| \cdots \| m_{t}, m_{i} \in V_{l}, i=1, \ldots, t$. Векторы $m_{i} \in V_{l}, i=1, \ldots, t$, называются блоками сообщения M. Алгоритм вычисления хэш-кода $h=H(M)$ итеративной функции хэширования состоит из последовательного вычисления значений так называемой функиии сжсатия (compression function) $g: V_{q} \times V_{l} \rightarrow V_{q}$ от результата предыдущей итерации и очередного блока сообщения:

$$
h_{i}=g\left(h_{i-1}, m_{i}\right), i=1, \ldots, t,
$$

где $h_{0}$ равно заданному фиксированному значению из множества $V_{q}$. Значение $h_{0}$ принято называть инициализационным вектором (initialization vector, initial value, IV). Значение хэш-кода $h$ полагается равным $f\left(h_{t}, \bar{M}\right)$, где $f: V_{q} \times V^{*} \rightarrow V_{n}-$ некоторое заключительное преобразование. Завершающее преобразование $f$ часто не зависит от $\bar{M}$ и является тождественным преобразованием первого аргумента (в этом случае $q=n$ ) или операцией редуцирования первого аргумента (в этом случае $q>n$ ). Всюду далее рассматриваем случай $q=n$. Для хэш-функции ГОСТ Р 34.11-94 завершающее преобразование заключается в применении функции сжатия к блоку, являющемуся суммой по модулю $2^{256}$ всех блоков сообщения: $f\left(h_{t}, \bar{M}\right)=g\left(h_{t}, \Sigma\right)$, $\Sigma=m_{1} \boxplus \ldots \boxplus m_{t}$. Данный подход эквивалентен дополнению исходного сообщения помимо двоичной записи длины значением $\Sigma$, которое принято называть контрольной суммой блоков сообщения.

Для удобства последующего изложения введем отображения $G_{i}^{\left(h_{0}\right)}$ : $V_{i l} \rightarrow V_{n}, i \in \mathbb{N}$, равенством

$$
G_{i}^{\left(h_{0}\right)}\left(m_{1}\left\|m_{2}\right\| \ldots \| m_{i}\right)=g\left(\left(\ldots g\left(g\left(h_{0}, m_{1}\right), m_{2}\right) \ldots\right), m_{i}\right),
$$


т. е. $G_{i}^{\left(h_{0}\right)}\left(m_{1}\left\|m_{2}\right\| \ldots \| m_{i}\right)=h_{i}$. Заметим, что $G_{1}^{(h)}(m)=g(h, m)$.

\section{2. Задачи, решаемые при криптографическом анализе функций хэширования}

Задачи, решаемые при криптографическом анализе функций хэширования, непосредственно следуют из криптографических требований к ним, определяемых сферами применения хэш-функций.

Традиционно криптографическая стойкость функции хэширования определяется трудоемкостью решения следующих основных задач.

1. Обращзение хэщ-функциии (построение прообраза, preimage attack). По заданному значению $h \in V_{n}$ найти такое сообщение $M \in V^{*}$, что $H(M)=h$.

2. Построение коллизии. Найти два различных сообщения $M, M^{\prime} \in V^{*}$, удовлетворяющих условию $H(M)=H\left(M^{\prime}\right)$.

Неупорядоченная пара различных сообщений $\left\{M, M^{\prime}\right\}$, для которой $H(M)=H\left(M^{\prime}\right)$, называется коллизией функции $H$.

3. Построение второго прообраза (second preimage attack). По заданному сообщению $M \in V^{*}$ найти такое отличное от $M$ сообщение $M^{\prime} \in V^{*}$, что $H\left(M^{\prime}\right)=H(M)$.

Заметим, что задачи 1-3 всегда имеют решение ввиду того, что функция хэширования не может являться биективным отображением. Для любой функции хэширования задачи 1 и 3 могут быть решены с использованием порядка $2^{n}$ операций, задача 2 - с использованием (в среднем) порядка $2^{\frac{n}{2}}$ операций, где $n-$ длина хэш-кода. Здесь под одной операцией понимается операция вычисления значения хэш-функции для одного сообщения, состоящего из одного блока.

Отметим, что при проведении криптографического анализа функции хэширования следует искать решения задач 1-3 не только непосредственно для самой функции хэширования, но и для функций сжатия, на основе которых она построена.

В ряде случаев представляет интерес решение следующей задачи.

4. Построение мультиколлизии (построение $r$-коллизии). Найти $r$ таких попарно различных сообщений $M_{1}, \ldots, M_{r} \in V^{*}$, что $H\left(M_{1}\right)=\ldots=$ $=H\left(M_{r}\right)$. 
Неупорядоченный набор попарно различных сообщений $\left\{M_{1}, \ldots, M_{r}\right\}$, для которого $H\left(M_{1}\right)=\ldots=H\left(M_{r}\right)$, называется мультиколлизией ( $r$-коллизией) функции $H$.

Построение мультиколлизии может являться промежуточным этапом методов анализа, решающих классические задачи анализа функций хэширования (см., например, метод построения коллизии, изложенный в § 6).

\section{3. Краткое описание хэш-функции ГОСТ Р 34.11-94}

Пусть $M$ - исходное сообщение, $\bar{M}=M_{1}\|\ldots\| M_{t}$ - сообщение, дополненное согласно стандарту ГОСТ Р 34.11-94 до длины, кратной 256, $M_{i} \in V_{256}, i=1, \ldots, t$. Пусть $H_{0}=I V-$ инициализационный вектор, $\Sigma=M_{1} \boxplus \ldots \boxplus M_{t},|M|$ - длина исходного сообщения $M$ в битах, представленная в виде двоичного вектора из $V_{256}$. Тогда вычисление хэш-кода $H(M)$ происходит следующим образом:

$$
\begin{gathered}
H_{i}=g\left(H_{i-1}, M_{i}\right) \quad i=1, \ldots, t, \\
H_{t+1}=g\left(H_{t},|M|\right), \\
H(M)=g\left(H_{t+1}, \Sigma\right),
\end{gathered}
$$

где $g: V_{256} \times V_{256} \rightarrow V_{256}-$ функция сжатия.

Значение функции сжатия $g(h, m), h=h_{3}\left\|h_{2}\right\| h_{1} \| h_{0}, h_{i} \in V_{64}$ $(i=0, \ldots, 3), m \in V_{256}$, вычисляется следующим образом:

$$
g(h, m)=\psi^{61}(h) \oplus \psi^{62}(m) \oplus \psi^{74}(s),
$$

где $\psi: V_{256} \rightarrow V_{256}$ - биективное линейное преобразование, $s=s_{3}\left\|s_{2}\right\| s_{1} \|$ $s_{0}, s_{i}=E\left(L_{i}(h, m), h_{i}\right) \in V_{64}, L_{i}: V_{256} \times V_{256} \rightarrow V_{256}$ - аффинные преобразования, $i \in\{0, \ldots, 3\}, E(K, P)-$ результат зашифрования открытого текста $P \in V_{64}$ с помощью ключа $K \in V_{256}$ алгоритмом ГОСТ 28147-89. Для последующего изложения не требуется дальнейшая детализация описания хэшфункции ГОСТ Р 34.11-94, заметим лишь, что преобразование $L_{0}$ при фиксированном первом аргументе является биективным преобразованием второго аргумента.

\section{4. Методы построения мультиколлизий для хэш-функций}

Как правило, трудоемкость алгоритмов построения мультиколлизий для функций хэширования является довольно высокой и не допускает их 
практическую реализацию. В то же время они имеют несомненную ценность с теоретической точки зрения, так как характеризуют конструкцию Меркля - Дамгорда как не идеальную и изменяют наш взгляд на теоретическую стойкость хэш-функций, используемых на практике. Принципы, лежащие в основе методов построения мультиколлизий, часто используются при криптографическом анализе конкретных хэш-функций.

Мультиколлизия ( $r$-коллизия) произвольной хэш-функции $H: V^{*} \rightarrow V_{n}$ может быть построена с использованием парадокса дней рождения с трудоемкостью порядка $(r !)^{\frac{1}{r}} \cdot 2^{\frac{n(r-1)}{r}}$ операций вычисления значения функции $H$ (см., например, $[1,5])$.

Далее рассмотрим функции хэширования, построенные по итеративному принципу, процедура дополнения сообщений которых имеет классический вид: исходное сообщение дополняется необходимым количеством нулей и единиц, а также двоичной записью длины исходного сообщения. Для таких функций хэширования существуют методы построения мультиколлизии при $r \geq 4$, более эффективные, чем метод, основанный на парадоксе дней рождения.

Впервые такой метод предложил А. Joux в работе [10]. Этот метод имеет трудоемкость порядка $\left\lceil\log _{2} r\right\rceil 2^{\frac{n}{2}}$ операций вычисления значения функции сжатия.

Если функция сжатия $g$ допускает эффективное построение неподвижных точек (т. е. нахождение такого блока сообщения $m \in V_{l}$ для заданного $h \in V_{n}$, что $\left.g(h, m)=h\right)$, то мультиколлизия для функции хэширования может быть построена с трудоемкостью порядка $3 \cdot 2^{\frac{n}{2}}$ операций вычисления значения функции сжатия и с использованием порядка $n \cdot 2^{\frac{n}{2}}$ бит памяти [12].

Некоторое время в качестве одного из способов противодействия методам построения мультиколлизий рассматривалось дополнение исходного сообщения различного рода контрольными суммами по примеру хэш-функции ГОСТ Р 34.11-94. Такой подход использовался при разработке хэш-функций MAELSTROM-0 [7], F-HASH [13] и конструкции 3C [8]. Однако в работе [9] были предложены эффективные методы построения мультиколлизий для таких хэш-функций. В частности, для хэш-функций, использующих контрольную сумму аналогично хэш-функции ГОСТ Р 34.11-94, описанный в [9] метод построения мультиколлизии ( $r$-коллизии) имеет трудоемкость порядка $\left(\frac{n^{2}}{2}+\left\lceil\log _{2} r\right\rceil\right) \cdot 2^{\frac{n}{2}}$ операций вычисления значения функции сжатия.

Предложим следующий очевидный, но не обнаруженный нами в публикациях метод построения мультиколлизии для функций хэширования, заключительное преобразование которых реализует вычисление значения функции 
сжатия от суммы всех блоков сообщения. Пусть завершающее преобразование $f$ хэш-функции Меркля-Дамгорда (см. § 1) реализовано следующим образом:

$$
f\left(h_{t}, \bar{M}\right)=g\left(h_{t}, m_{1} \boxplus m_{2} \boxplus \ldots\right),
$$

где $m_{i}$ - блоки исходного сообщения, $i=1,2, \ldots$ Пусть $\boxminus-$ операция вычитания по модулю $2^{n}$. Определим функцию $g^{\prime}: V_{n} \times V_{l} \rightarrow V_{n}$ равенством $g^{\prime}(h, m)=g(g(h, m), \boxminus m), h \in V_{n}, m \in V_{l}$. С помощью метода, основанного на парадоксе дней рождения, последовательно строим коллизии $\left(m_{1}^{(0)}, m_{1}^{(1)}\right),\left(m_{2}^{(0)}, m_{2}^{(1)}\right), \ldots,\left(m_{\left\lceil\log _{2} r\right\rceil}^{(0)}, m_{\left\lceil\log _{2} r\right\rceil}^{(1)}\right)$, так что $g^{\prime}\left(h_{2 i-2}, m_{i}^{(0)}\right)=$ $=g^{\prime}\left(h_{2 i-2}, m_{i}^{(1)}\right)=h_{2 i}, i=1, \ldots,\left\lceil\log _{2} r\right\rceil$. Мультиколлизию $(r$-коллизию) составляют любые $r$ попарно различных сообщений вида $m_{1}^{\left(i_{1}\right)}\left\|\left(\boxminus m_{1}^{\left(i_{1}\right)}\right)\right\|$ $m_{2}^{\left(i_{2}\right)}\left\|\left(\boxminus m_{2}^{\left(i_{2}\right)}\right)\right\| \ldots\left\|m_{\left\lceil\log _{2} r\right\rceil}^{\left(i_{1}\right)}\right\|\left(\boxminus m_{\left\lceil\log _{2} r\right\rceil}^{\left(i_{1}\right)}\right), i_{j}=0,1, j=1, \ldots,\left\lceil\log _{2} r\right\rceil$ (всего таких сообщений $2^{\left[\log _{2} r\right\rceil}$ ). Трудоемкость предложенного метода составляет порядка $\left\lceil\log _{2} r\right\rceil \cdot 2^{\frac{n}{2}+1}$ операций вычисления значения функции сжатия, что при $n>\sqrt{2\left\lceil\log _{2} r\right\rceil}$ меньше трудоемкости метода работы [9] и всего лишь в два раза превосходит трудоемкость метода [10] построения мультиколлизии для хэш-функций, не использующих контрольные суммы.

Отметим, что по эффективному методу поиска мультиколлизии можно строить эффективные методы поиска второго прообраза и (второго) мультипрообраза функции хэширования (см. $[9,11,12])$.

\section{5. Метод Вагнера решения обобщенной «задачи о днях рождения»}

Рассмотрим обобщенную «задачу о днях рождения» [19]. Пусть $a \in V_{n}$ и $L_{1}, \ldots, L_{2^{k}}$ - независимые равновероятные выборки из множества $V_{n}$, причем $k+1$ делит $n$ и $\left|L_{1}\right|=\ldots=\left|L_{2^{k}}\right|=2^{\frac{n}{k+1}}$. Требуется найти такие $x_{1} \in L_{1}, \ldots, x_{2^{k}} \in L_{2^{k}}$, что

$$
x_{1}+\ldots+x_{2^{k}}=a
$$

где $+\in\{\oplus, \boxplus\}$.

В [19] предложен метод нахождения таких векторов со средней трудоемкостью порядка $2^{\frac{n}{k+1}+k}$ операций сложения векторов из $V_{n}$ и объемом необходимой памяти порядка $n \cdot 2^{\frac{n}{k+1}+k}$ бит.

Покажем, что метод Вагнера может быть использован для построения одновременно нескольких коллизий хэш-функций, использующих контрольную сумму аналогично хэш-функции ГОСТ Р 34.11-94. Пусть завершающее преобразование $f$ хэш-функции Меркля-Дамгорда (см. §1) реализовано 
следующим образом:

$$
f\left(h_{t}, \bar{M}\right)=g\left(h_{t}, m_{1} \boxplus m_{2} \boxplus \ldots\right),
$$

где $m_{i}-$ все блоки исходного сообщения, $i=1,2, \ldots$

Пусть для любого $h \in V_{n}$ существует метод построения коллизии функции $G_{s}^{(h)}$ (при некотором $s=1,2, \ldots$ ) с трудоемкостью, равной $Q$ операциям вычисления значения функции сжатия. В общем случае таким методом может являться метод, основанный на парадоксе дней рождения для функции сжатия, т. е. при $s=1$.

Зафиксируем $k \in \mathbb{N}$ - параметр метода. Построим $N=\frac{n}{k+1} 2^{k}$ последовательных коллизий $\left(M_{i}^{(0)}, M_{i}^{(1)}\right), i=1, \ldots, N$, функции $G_{s}^{(h)}$ :

$$
G_{s}^{\left(h_{i-1}\right)}\left(M_{i}^{(0)}\right)=G_{s}^{\left(h_{i-1}\right)}\left(M_{i}^{(1)}\right)=h_{i}, \quad i=1, \ldots, N .
$$

Для построения мультиколлизии хэш-функции $H$ достаточно среди сообщений вида

$$
M_{1}^{\left(\varepsilon_{1}\right)}\|\ldots\| M_{N}^{\left(\varepsilon_{N}\right)}, \quad\left(\varepsilon_{1}, \ldots, \varepsilon_{N}\right) \in V_{N},
$$

выбрать сообщения с одинаковыми значениями суммы блоков по модулю $2^{n}$. Применим для этого метод Вагнера [19], исходными списками для которого являются

$$
L_{j}=\left\{\sum_{l=\frac{n}{k+1}(j-1)+1}^{\frac{n}{k+1} j} \varepsilon_{l}\left(\widetilde{M}_{l}^{(0)} \boxminus \widetilde{M}_{l}^{(1)}\right) \mid\left(\varepsilon_{\frac{n}{k+1}}(j-1)+1, \ldots, \varepsilon_{\frac{n}{k+1} j}\right) \in V_{\frac{n}{k+1}}\right\}, \quad j=1, \ldots, 2^{k},
$$

где $\widetilde{M}_{l}^{(t)}$ - сумма по модулю $2^{n}$ блоков сообщения $M_{l}^{(t)}, t=0,1, l=1, \ldots, N$.

В результате применения метода Вагнера с вероятностью, близкой к единице (см. [19]), будет построен такой ненулевой вектор $\left(\varepsilon_{1}, \ldots, \varepsilon_{N}\right) \in V_{N}$, что

$$
\sum_{l=1}^{N} \varepsilon_{l}\left(\widetilde{M}_{l}^{(0)} \boxminus \widetilde{M}_{l}^{(1)}\right)=0 .
$$

Тогда одинаковые значения хэш-кода будут иметь пары сообщений

$$
R_{\alpha}^{(0)}=M_{1}^{\left(\alpha_{1}\right)}\|\ldots\| M_{N}^{\left(\alpha_{N}\right)} \quad \text { и } \quad R_{\alpha}^{(1)}=M_{1}^{\left(\alpha_{1}+\varepsilon_{1}\right)}\|\ldots\| M_{N}^{\left(\alpha_{N}+\varepsilon_{N}\right)},
$$

где $\alpha=\left(\alpha_{1}, \ldots, \alpha_{N}\right) \in V_{N}$ и $\alpha_{l}=0$, если $\varepsilon_{l}=1$ и $\alpha_{l} \in\{0,1\}$, если $\varepsilon_{l}=0$. Количество построенных коллизий равняется $2^{\tau}$, где $\tau=\sum_{l=1}^{N}\left(1-\varepsilon_{l}\right)$ (здесь производится целочисленное суммирование). В предположении о независимости 
и равновероятности распределения случайных величин $\varepsilon_{l}, l=1, \ldots, N$, случайная величина $\tau$ имеет биномиальное распределение и ее среднее равняется $\frac{N}{2}$. Таким образом, с помощью описанного метода с трудоемкостью порядка $Q N+2^{\frac{n}{k+1}+k}$ операций вычисления значения функции сжатия может быть конструктивно описано в среднем не менее $2^{\frac{N}{2}}$ коллизий. При этом значения контрольных сумм блоков сообщений, а следовательно, и значения хэшкодов двух различных пар сообщений, образующих коллизии, вообще говоря, различны, т. е. построенные пары коллизий не образуют мультиколлизию.

\section{6. Методы построения коллизий и прообразов для хэш-функции ГОСТ Р 34.11-94}

Рассмотрим методы построения коллизий и прообразов для хэшфункции ГОСТ Р 34.11-94, предложенные в работах [14] и [15]. Мы излагаем наше понимание тех этапов этих методов, описание которых опущено в указанных работах.

Часть результатов, излагаемых ниже, анонсирована в [2].

\section{1. Построение коллизии функции сжатия хэш-функции ГОСТ P 34.11-94}

Пусть $g(h, m)$ - функция сжатия алгоритма ГОСТ Р 34.11-94, значение $h=h_{3}\left\|h_{2}\right\| h_{1} \| h_{0}$ фиксировано, $h_{i} \in V_{64}(i=0,1,2,3), h_{0}=h_{0}^{(1)} \| h_{0}^{(0)}$, $h_{0}^{(1)}, h_{0}^{(0)} \in V_{32}$ и $h_{0}^{(1)}=h_{0}^{(0)}$. Так как $\psi-$ биективное линейное преобразование, то из (1) следует, что если $\widehat{h}=g(h, m)$, то существуют такие линейные преобразования $L^{x}, L^{y}, L^{z}: V_{256} \rightarrow V_{256}$, что

$$
L^{x}(\widehat{h})=L^{y}(h) \oplus L^{z}(m) \oplus s .
$$

Если $L^{x}(\widehat{h})=\widehat{h_{3}}\left\|\widehat{h_{2}}\right\| \widehat{h_{1}} \| \widehat{h_{0}}, \widehat{h_{i}} \in V_{64}(i=0,1,2,3)$, то существуют такие линейные преобразования $L_{i}^{y}, L_{i}^{z}: V_{256} \rightarrow V_{64}(i=0,1,2,3)$, что

$$
\widehat{h_{i}}=L_{i}^{y}(h) \oplus L_{i}^{z}(m) \oplus E\left(L_{i}(h, m), h_{i}\right), \quad i=0,1,2,3 .
$$

Так как преобразование $L_{0}(h, m)$ при фиксированном значении $h$ является биективным по переменной $m$, то в равенстве (3) при $i=0$ можно сделать замену переменной $m \rightarrow k$, где $k=L_{0}(h, m)$. Для соответствующего линейного преобразования $L_{0}^{h}(k)$ получаем:

$$
\widehat{h_{0}}=L_{0}^{y}(h) \oplus L_{0}^{h}(k) \oplus E\left(k, h_{0}\right) .
$$


Пусть $k=k_{1} \| k_{0}, k_{0}, k_{1} \in V_{128}$, и $d \in V_{64}-$ некоторый фиксированный вектор. Тогда из равенства $L_{0}^{h}(k)=d$ следует выполнение равенств

$$
\left\{\begin{array}{l}
L_{0}^{h, 0}\left(k_{0}\right)=d_{0}, \\
L_{0}^{h, 1}\left(k_{1}\right)=d \oplus d_{0}
\end{array}\right.
$$

для некоторого $d_{0} \in V_{64}$ при однозначно определенных аффинных отображениях $L_{0}^{h, 0}, L_{0}^{h, 1}: V_{128} \rightarrow V_{64}$.

Опишем теперь метод построения коллизии функции сжатия алгоритма ГОСТ Р 34.11-94, предложенный в [15].

1. Случайно выбираем различные векторы $d_{0}^{(i)}, 1 \leq i \leq 2^{32}$, и для каждого $i$ выполняем шаги 2-4.

2. Находим $2^{64}$ решений уравнения $L_{0}^{h, 0}\left(k_{0}\right)=d_{0}^{(i)}$ и каждое решение $k_{0}^{(j)}, 1 \leq j \leq 2^{64}$, записываем в память по адресу $P$, где $P-$ результат зашифрования открытого текста $h_{0}$ с использованием первых четырех итераций алгоритма ГОСТ 28147-89 (итераций с номерами 1-4 из 32 итераций полного алгоритма) и ключа $k_{0}^{(j)}$.

3. Находим $2^{64}$ решений уравнения $L_{0}^{h, 1}\left(k_{1}\right)=d \oplus d_{0}^{(i)}$. Для каждого решения $k_{1}^{(j)}, 1 \leq j \leq 2^{64}$, вычисляем значение $P^{\prime}$, равное результату расшифрования информационного блока $h_{0}$ с использованием четырех итераций алгоритма ГОСТ 28147-89 (итераций с номерами 5-8 из 32 итераций полного алгоритма) и ключа $k_{1}^{(j)}$. К хранящейся в памяти по адресу $P^{\prime}$ информации дописываем значение $k_{1}^{(j)}$.

4. Для каждого 256-битного вектора $\left(k_{1}^{(j)}, k_{0}^{(j)}\right)$, образовавшегося после выполнения шагов 2-3, вычисляем соответствующее значение $m_{j}^{(i)}$, $1 \leq j \leq 2^{64}$.

5. Для каждых $i=1, \ldots, 2^{32}$ и $j=1, \ldots, 2^{64}$ вычисляем

$$
\widehat{h}_{l}^{(j, i)}=L_{l}^{y}(h) \oplus L_{l}^{z}\left(m_{j}^{(i)}\right) \oplus E\left(L_{l}\left(h, m_{j}^{(i)}\right), h_{l}\right), \quad l=1,2,3 .
$$

Заметим, что значение $\widehat{h}_{0}^{(j, i)}$ одинаково для всех $i=1, \ldots, 2^{32}$ и $j=$ $=1, \ldots, 2^{64}$.

6. Осуществляем поиск двух таких пар $(i, j) \neq\left(i^{\prime}, j^{\prime}\right),(i, j),\left(i^{\prime}, j^{\prime}\right) \in$ $\in\left\{1, \ldots, 2^{32}\right\} \times\left\{1, \ldots, 2^{64}\right\}$, что

$$
\widehat{h}_{1}^{(j, i)}=\widehat{h}_{1}^{\left(j^{\prime}, i^{\prime}\right)}, \quad \widehat{h}_{2}^{(j, i)}=\widehat{h}_{2}^{\left(j^{\prime}, i^{\prime}\right)}, \quad \widehat{h}_{3}^{(j, i)}=\widehat{h}_{3}^{\left(j^{\prime}, i^{\prime}\right)} .
$$


Если такая пара $(i, j),\left(i^{\prime}, j^{\prime}\right)$ найдена, то $g\left(h, m_{j}^{(i)}\right)=g\left(h, m_{j^{\prime}}^{\left(i^{\prime}\right)}\right)$ и коллизия для функции сжатия построена.

Трудоемкость второго, третьего и четвертого этапов изложенного метода не превосходит по порядку $2^{64}$ операций вычисления функции сжатия алгоритма ГОСТ Р 34.11-94, при этом требуется порядка $2^{64} \cdot 40 \approx 2^{70}$ байт памяти. Трудоемкость пятого и шестого этапов метода составляет порядка $2^{96}$ операций вычисления функции сжатия алгоритма ГОСТ Р 34.11-94.

Оценим объем памяти, необходимой для реализации пятого и шестого этапов метода, и предложим конкретизацию этих этапов, направленную на уменьшение требуемого объема памяти. Объем памяти, используемой на пятом и шестом этапах метода, определяется необходимостью построения коллизии функции вида $F: V_{256} \rightarrow V_{192}, F(m)=\widehat{h}_{3}(m)\left\|\widehat{h}_{2}(m)\right\| \widehat{h}_{1}(m)$, на блоках $m_{j}^{(i)} \in V_{256}$ с одинаковыми для данной функции $F$ значениями $\widehat{h}_{0}\left(m_{j}^{(i)}\right)$. Для этого требуется память из $2^{96}$ ячеек. Для каждого $m_{j}^{(i)}$ младшие 96 бит значения $F\left(m_{j}^{(i)}\right)$ являются адресом ячейки, в которую записываются старшие 96 бит вместе с соответствующим 64-битным значением $d_{0}^{(i)}$ (это выгоднее, чем хранить само 256-битное значение $m_{j}^{(i)}$, которое после обнаружения коллизии может быть восстановлено по $d_{0}^{(i)}$ с пренебрежимо малой в данном случае трудоемкостью порядка $2^{64}$ вычислений функции сжатия). Если при повторном обращении к ячейке старшие 96 бит совпали с хранящимися в ней битами, то, очевидно, обнаружена коллизия $F$, и мы восстанавливаем ее с помощью соответствующих значений $d_{0}^{(i)}$, иначе перезаписываем содержимое ячейки. Таким образом, объем памяти, необходимый для реализации метода построения коллизии функции сжатия, составляет не менее $2^{96} \cdot 160$ бит (5. $2^{98}$ байт).

\section{2. Построение коллизии хэш-функции ГОСТ Р 34.11-94}

Пусть $H_{0}=I V-$ произвольный инициализационный вектор. Метод построения коллизии хэш-функции ГОСТ Р 34.11-94 с инициализационным вектором $H_{0}$ состоит из следующих этапов.

1. Для каждого $i=1, \ldots, 512$ последовательно выполняем шаги 2-3.

2. Имеем значение $H_{2 i-2}$. Случайным образом выбираем векторы $M_{2 i-1} \in$ $\in V_{256}$ и для каждого вычисляем $H_{2 i-1}=g\left(H_{2 i-2}, M_{2 i-1}\right)$ до тех пор, пока не найдем такой вектор $M_{2 i-1} \in V_{256}$, что $H_{2 i-1}=h\left\|h_{0}^{(1)}\right\| h_{0}^{(0)}$, $h \in V_{192}, h_{0}^{(1)}, h_{0}^{(0)} \in V_{32}$ и $h_{0}^{(1)}=h_{0}^{(0)}$. 
3. Имеем $H_{2 i-1}$. С использованием описанного в п. 6.1 метода находим такие $M_{2 i}^{(0)}, M_{2 i}^{(1)} \in V_{256}$, что $M_{2 i}^{(0)} \neq M_{2 i}^{(1)}$ и $g\left(H_{2 i-1}, M_{2 i}^{(0)}\right)=$ $g\left(H_{2 i-1}, M_{2 i}^{(1)}\right)=H_{2 i}$.

4. Формируем списки $L_{0}, L_{1}, L_{2}, L_{3}$. Список $L_{j}, 0 \leq j \leq 3$, состоит из всех векторов $\left(\varepsilon_{128}, \ldots, \varepsilon_{1}\right) \in V_{128}$, для которых

$$
\begin{aligned}
2^{64} \mid\left(\sum_{l=1}^{64} \varepsilon_{l}\left(M_{2 l+256 j}^{(0)} \boxminus M_{2 l+256 j}^{(1)}\right) \boxminus\right. & \\
& \left.\boxminus \sum_{l=1}^{64} \varepsilon_{l+64}\left(M_{2 l+256 j+128}^{(0)} \boxminus M_{2 l+256 j+128}^{(1)}\right)\right) .
\end{aligned}
$$

Средняя мощность списков $L_{j}, 0 \leq j \leq 3$, составляет порядка $2^{64}$.

5. Формируем списки $L_{0}^{\prime}, L_{1}^{\prime}$. Список $L_{j}^{\prime}, j=0,1$, состоит из всех векторов $\left(\varepsilon_{256}, \ldots, \varepsilon_{1}\right) \in V_{256}$, для которых выполняются условия

$$
\left\{\begin{aligned}
& 2^{128} \mid {\left[\left(\sum_{l=1}^{128} \varepsilon_{l}\left(M_{2 l+256 j}^{(0)} \boxminus M_{2 l+256 j}^{(1)}\right)\right) \boxminus\right.} \\
&\left.\boxminus\left(\sum_{l=1}^{128} \varepsilon_{l+128}\left(M_{2 l+256 j+256}^{(0)} \boxminus M_{2 l+256 j+256}^{(1)}\right)\right)\right], \\
&\left(\varepsilon_{128}, \ldots, \varepsilon_{1}\right) \in L_{2 j}, \quad\left(\varepsilon_{256}, \ldots, \varepsilon_{129}\right) \in L_{2 j+1} .
\end{aligned}\right.
$$

Средняя мощность списков $L_{j}^{\prime}, 0 \leq j \leq 1$, составляет порядка $2^{64}$.

6. Осуществляем поиск такого ненулевого вектора $\left(\varepsilon_{512}, \ldots, \varepsilon_{1}\right) \in V_{256}$, что

$$
\left\{\begin{array}{l}
\sum_{l=1}^{512} \varepsilon_{l}\left(M_{2 l}^{(0)} \boxminus M_{2 l}^{(1)}\right)=0, \\
\left(\varepsilon_{256}, \ldots, \varepsilon_{1}\right) \in L_{0}^{\prime}, \quad\left(\varepsilon_{512}, \ldots, \varepsilon_{257}\right) \in L_{1}^{\prime} .
\end{array}\right.
$$

Если такой вектор $\left(\varepsilon_{512}, \ldots, \varepsilon_{1}\right)$ найден, то пары сообщений $M$ и $M^{\prime}$,

$$
\begin{gathered}
M=M_{1024}^{\left(\alpha_{512}\right)}\left\|M_{1023}\right\| \ldots\left\|M_{2}^{\left(\alpha_{1}\right)}\right\| M_{1}, \\
M^{\prime}=M_{1024}^{\left(\alpha_{512} \oplus \varepsilon_{512}\right)}\left\|M_{1023}\right\| \ldots\left\|M_{2}^{\left(\alpha_{1} \oplus \varepsilon_{1}\right)}\right\| M_{1},
\end{gathered}
$$

образуют коллизию хэш-функции ГОСТ Р 34.11-94. Здесь $\alpha_{l}=0$, если $\varepsilon_{l}=1$, и $\alpha_{l} \in\{0,1\}$, если $\varepsilon_{l}=0, l=1, \ldots, 512$. 
Трудоемкость второго этапа метода в среднем составляет порядка $2^{32}$ операций вычисления функции сжатия алгоритма ГОСТ Р 34.11-94. Трудоемкость третьего этапа метода составляет порядка $2^{96}$ операций вычисления функции сжатия алгоритма ГОСТ Р 34.11-94, при этом требуется не менее $5 \cdot 2^{98}$ байт памяти.

Следует отметить, что описание шагов 4-6 в работах [14] и [15] отсутствует, но имеется только ссылка на работу [19]. Представленное здесь описание шагов 4-6 является нашим применением метода Вагнера (см. §5) для построения коллизии. Отметим также, что, как показано в §5, описанный метод приводит к конструктивному описанию в среднем не менее $2^{256}$ различных коллизий хэш-функции ГОСТ Р 34.11-94.

Общая трудоемкость метода построения коллизий для алгоритма ГОСТ Р 34.11-94 составляет порядка $2^{105}$ операций вычисления функции сжатия алгоритма ГОСТ Р 34.11-94, при этом требуется $5 \cdot 2^{98}$ байт памяти.

\section{3. Построение прообраза для функции сжатия хэш-функции ГОСТ P 34.11-94}

Пусть значения $h, \widehat{h} \in V_{256}$ фиксированы, $h=h_{3}\left\|h_{2}\right\| h_{1}\left\|h_{0}, \widehat{h}=\widehat{h}_{3}\right\| \widehat{h}_{2} \|$ $\widehat{h}_{1}\left\|\widehat{h}_{0}, h_{i}, \widehat{h}_{i} \in V_{64}(0 \leq i \leq 3), h_{0}=h_{0}^{(1)}\right\| h_{0}^{(0)}, h_{0}^{(1)}, h_{0}^{(0)} \in V_{32}$ и $h_{0}^{(1)}=h_{0}^{(0)}$.

Используя обозначения п. 6.1, алгоритм построения прообраза для функции сжатия (решения уравнения $g(h, m)=\widehat{h}$ относительно $m$ ) можно описать следующим образом.

1. Перебираем все различные векторы $d_{0}^{(i)} \in V_{64}, 1 \leq i \leq 2^{64}$, и для каждого $i$ выполняем шаги 2-4.

2. Находим $2^{64}$ решений уравнения $L_{0}^{h, 0}\left(k_{0}\right)=d_{0}^{(i)}$ и каждое решение $k_{0}^{(j)}$, $1 \leq j \leq 2^{64}$, записываем в память по адресу $P$, где $P-$ результат зашифрования открытого текста $h_{0}$ с использованием четырех итераций алгоритма ГОСТ 28147-89 и ключа $k_{0}^{(j)}$.

3. Находим $2^{64}$ решений уравнения $L_{0}^{h, 1}\left(k_{1}\right)=d \oplus d_{0}^{(i)}$. Для каждого решения $k_{1}^{(j)}, 1 \leq j \leq 2^{64}$, вычисляем значение $P^{\prime}$, равное результату расшифрования информационного блока $h_{0}$ с использованием четырех итераций алгоритма ГОСТ 28147-89 и ключа $k_{1}^{(j)}$. К хранящейся в памяти по адресу $P^{\prime}$ информации дописываем значение $k_{1}^{(j)}$.

4. Для каждого 256-битного вектора $\left(k_{1}^{(j)}, k_{0}^{(j)}\right)$, образовавшегося после выполнения шагов 2-3, вычисляем соответствующее значение $m_{j}^{(i)}$, $1 \leq j \leq 2^{64}$. 
5. Для каждых $i, j=1, \ldots, 2^{64}$ вычисляем

$$
\widehat{h}_{l}^{(j, i)}=L_{l}^{y}(h) \oplus L_{l}^{z}\left(m_{j}^{(i)}\right) \oplus E\left(L_{l}\left(h, m_{j}^{(i)}\right), h_{l}\right), \quad l=1,2,3,
$$

и проверяем выполнение равенств

$$
\widehat{h}_{l}^{(j, i)}=\widehat{h}_{l}, \quad l=1,2,3 .
$$

Заметим, что $\widehat{h}_{0}^{(j, i)}=\widehat{h}_{0}$ для всех $i, j=1, \ldots, 2^{64}$. Если равенства (6) выполнены для некоторых $(j, i)$, то $g\left(h, m_{j}^{(i)}\right)=\widehat{h}$ и прообраз функции сжатия построен.

Трудоемкость второго, третьего и четвертого этапов изложенного метода не превосходит по порядку $2^{64}$ операций вычисления функции сжатия алгоритма ГОСТ Р 34.11-94, при этом требуется $2^{64} \cdot 40 \approx 2^{70}$ байт памяти. Трудоемкость пятого этапа метода составляет порядка $2^{128}$ операций вычисления функции сжатия алгоритма ГОСТ Р 34.11-94, при этом искомый прообраз находится с вероятностью $2^{-64}$.

\section{4. Построение прообраза для хэш-функции ГОСТ Р 34.11-94}

Пусть $H_{0}=I V$ - произвольный инициализационный вектор и фиксировано некоторое значение $H \in V_{256}$. Метод построения прообраза хэш-кода $H$ состоит из следущих шагов.

1. Для каждого $j=1, \ldots, 256$ последовательно находим такие пары блоков сообщения $M_{j}^{(0)}, M_{j}^{(1)}$ и промежуточные значения хэш-функции $H_{j}$, что $M_{j}^{(0)} \neq M_{j}^{(1)}$ и $H_{j}=g\left(H_{j-1}, M_{j}^{(0)}\right)=g\left(H_{j-1}, M_{j}^{(1)}\right)$.

2. Методом случайного поиска находим такие $M_{257}^{(l)} \in V_{256}, l \in$ $\in\left\{1, \ldots, 2^{64}\right\}$, что

$$
g\left(g\left(H_{256}, M_{257}^{(l)}\right)\right)=H_{258}^{(l)},
$$

где $H_{258}^{(l)}=h_{3}^{(l)}\left\|h_{2}^{(l)}\right\| h_{1}^{(l)}\left\|h_{0}^{(l)}, h_{i}^{(l)} \in V_{64}(0 \leq i \leq 3), h_{0}^{(l)}=h_{0}^{(1, l)}\right\|$ $h_{0}^{(0, l)}, h_{0}^{(1, l)}, h_{0}^{(0, l)} \in V_{32}$ и $h_{0}^{(1, l)}=h_{0}^{(0, l)}, M_{258} \in V_{256}$ соответствует двоичному представлению числа $257 \cdot 256$.

3. Для каждого $l=1, \ldots, 2^{64}$ применяем описанный в п. 6.3. метод нахождения решения уравнения $g\left(H_{258}^{(l)}, \Sigma\right)=H$ относительно $\Sigma$. В среднем для одного значения $l$ решение данного уравнения будет найдено. Обозначим это решение через $\Sigma^{m}$, а соответствующий информационный блок $M_{257}^{(l)}$ через $M_{257}$. 
4. Находим вектор $\varepsilon=\left(\varepsilon_{256}, \ldots, \varepsilon_{1}\right) \in V_{256}$, удовлетворяющий условию

$$
M_{1}^{\left(\varepsilon_{1}\right)} \boxplus \ldots \boxplus M_{256}^{\left(\varepsilon_{256}\right)}=\Sigma^{m} \boxminus M_{257}
$$

Если такой вектор $\varepsilon$ найден, то сообщение $M_{257}\left\|M_{256}^{\left(\varepsilon_{256}\right)}\right\| \ldots \| M_{1}^{\left(\varepsilon_{1}\right)}$ является прообразом для хэш-кода $H$.

Нахождение коллизий функции сжатия на первом этапе может осуществляться с помощью метода, основанного на парадоксе дней рождения. Тогда трудоемкость первого этапа изложенного метода составляет порядка $2^{136}$ операций вычисления функции сжатия алгоритма ГОСТ Р 34.11-94. Трудоемкость второго этапа изложенного метода составляет порядка $2^{97}$ операций вычисления функции сжатия алгоритма ГОСТ Р 34.11-94. Трудоемкость третьего этапа изложенного метода составляет порядка $2^{192}$ операций вычисления функции сжатия алгоритма ГОСТ Р 34.11-94, при этом требуется порядка $2^{70}$ байт памяти. Трудоемкость последнего этапа изложенного метода составляет порядка $2^{128}$ операций вычисления функции сжатия алгоритма ГОСТ Р 34.11-94.

Общая трудоемкость метода построения прообраза для алгоритма ГОСТ Р 34.11-94 составляет порядка $2^{192}$ операций вычисления функции сжатия алгоритма ГОСТ Р 34.11-94, при этом требуется $2^{70}$ байт памяти.

\section{7. Метод построения коллизии хэш-функции ГОСТ Р 34.11-94 в условиях ограниченной памяти}

Метод построения коллизии, рассмотренный в 6 , требует использования значительного объема памяти. Предложим модификацию алгоритма в условиях ограниченной памяти и получим оценку минимального объема памяти, при котором трудоемкость модифицированного алгоритма меньше «универсальной» оценки.

Уменьшить объем используемой методом памяти без увеличения его трудоемкости могли бы позволить итерационные методы [18], но для их применения требуется легко вычисляемая функция $f: V_{192} \rightarrow V_{192}$, коллизия которой с большой вероятностью дает нужную коллизию $F$. Как построить такую функцию $f-$ не ясно. Однако можно уменьшить объем используемой памяти ценой увеличения трудоемкости. Опишем такую модификацию, т. е. способ построения коллизии функции $F$.

Пусть имеется память из $2^{w}$ ячеек по $192-w+64$ бит. Для каждого $m_{j}^{(i)}$ (верхняя граница значений $i$ теперь может быть другой) младшие ш бит 
значения $F\left(m_{j}^{(i)}\right)$ являются адресом ячейки, в которую записываются старшие 192 - бит вместе с соответствующим $d_{0}^{(i)}$. Если при повторном обращении к ячейке старшие 192 - ш бит совпадают с битами, содержащимися в ячейке, то, очевидно, обнаружена коллизия $F$, и мы восстанавливаем ее с помощью соответствующих значений $d_{0}^{(i)}$, иначе перезаписываем содержимое ячейки.

В качестве вероятностной модели для оценки трудоемкости алгоритма естественно использовать равновероятную выборку с возвращением из $n$ элементов с памятью на $z$ элементов [18]. В этой модели среднее число элементов, выбранных до первого повтора, при больших $n$ можно считать равным

$$
\sum_{k=0}^{z-1} e^{-k^{2} /(2 n)}+(n / z) e^{-z^{2} /(2 n)} .
$$

Трудоемкость алгоритма определяется трудоемкостью построения коллизий для 512 различных $F$. В нашем случае $n=2^{192}, z=2^{\text {w }}$, поэтому средняя трудоемкость модифицированного алгоритма приближенно равна

$$
512\left(\sum_{k=0}^{2^{w}-1} e^{-k^{2} / 2^{193}}+2^{192-w} e^{-2^{2 w-193}}\right)
$$

вычислений функции сжатия. Ее легко вычислять, аппроксимируя сумму интегралом:

$$
\sum_{k=0}^{z-1} e^{-k^{2} /(2 n)} \approx \int_{0}^{z} e^{-x^{2} /(2 n)} d x
$$

Легко увидеть, что средняя трудоемкость «универсального» метода построения коллизии хэш-функции ГОСТ Р 34.11-94 (параллельного метода поиска коллизий [18]) приближенно равна $3 \sqrt{\pi} \cdot 2^{128}$ вычислений значения функции сжатия. Появление в этом выражении множителя 3 объясняется тем, что из-за применения функции $g$ к блокам, состоящим из двоичной записи длины сообщения и контрольной суммы всех блоков сообщения, минимальное число вычислений значения функции сжатия, необходимое для вычисления значения итерируемой функции параллельного метода поиска коллизий, равно 3. Вычисления показывают, что трудоемкость модифицированного алгоритма меньше этой величины при $ш \geq 71$, т. е. при объеме памяти $2^{w}\lceil(192-w+64) / 8\rceil>2^{75}$ байт. 


\section{8. Выводы и нерешенные задачи}

В работе предложен новый метод построения мультиколлизии для функций хэширования, которые на завершающем этапе применяют функцию сжатия к сумме по модулю $2^{n}$ всех блоков сообщения (§4). При ряде значений параметров трудоемкость предложенного метода меньше трудоемкости предлагавшихся ранее методов построения мультиколлизий для хэш-функций такого типа.

Показано, что использование метода Вагнера при анализе функций хэширования, которые функцию сжатия применяют к сумме по модулю $2^{n}$ всех блоков сообщения, приводит к конструктивному описанию большого числа различных коллизий (§5).

Применение методов работ $[14,15]$ к хэш-функции ГОСТ Р 34.11-94 использует следующие ее особенности:

- использование в функции сжатия четырех параллельных реализаций блочного шифра;

- линейная зависимость информационных блоков, поступающих на вход четырех параллельных реализаций блочного шифра, от очередного блока сообщения и линейная зависимость значения функции сжатия от результатов зашифрования четырех параллельных реализаций блочного шифра и очередного блока сообщения;

- особенности алгоритма шифрования ГОСТ 28147-89 (возможность эффективного построения неподвижных точек).

Для метода построения коллизии хэш-функции ГОСТ Р 34.11-94, предложенного в $[14,15]$, показано, что объем необходимой памяти составляет не менее $5 \cdot 2^{98}$ байт (§6). Предложена модификация метода в условиях ограниченной памяти и показано, что трудоемкость модифицированного алгоритма меньше «универсальной» оценки, если объем памяти не меньше $2^{75}$ байт $(\S 7)$.

Представляет интерес решение следующих задач:

- определить точное значение вероятности успеха метода Вагнера и построить его эффективные модификации, направленные на уменьшение совокупной мощности списков;

- построить легко вычисляемую функцию $f: V_{192} \rightarrow V_{192}$ и итерационный метод, позволяющие уменьшить объем памяти, необходимой для построения коллизии функции сжатия хэш-функции ГОСТ Р 34.11-94. 
Авторы благодарят Зубкова А. М. и Тришина А. Е. за полезные замечания, которые помогли существенно улучшить изложение представленных в настоящей работе результатов.

\section{Список литературы}

1. Колчин В.Ф., Севастьянов Б.А., Чистяков В.П. Случайные размещения. - М.: Наука, 1976.

2. Матюхин Д.В., Шишкин В.А. О криптографической стойкости хэшфункции ГОСТ Р 24.11-94 // Обозр. прикл. и промыш. матем. - 2010. T. 17. - C. 750-751.

3. FIPS PUB 180-1. Secure hash standard. - April, 1995. http://www.itl.nist.gov/fipspubs/fip180-1.htm.

4. FIPS PUB 180-2. Secure hash standard. - August, 2002. http://csrc.nist.gov/publications/fips/fips180-2/fips180-2.pdf

5. Aumasson J.-P. Faster multicollisions // INDOCRYPT'08. Lect. Notes Comput. Sci. - 2008. - V. 5365. - P. 67-77.

6. Damgaard I. A design principle for hash functions // CRYPTO'89. Lect. Notes Comput. Sci. - 1990. - V. 435. - P. 416-427.

7. Filho D. G., Barreto P., Rijmen $V$. The Maelstrom-0 hash function. - 6th Brazilian Symp. Inf. Comput. Syst. Security, 2006.

8. Gauravaram P. Cryptographic Hash Functions: Cryptanalysis, Design and Applications. PhD thesis, Queensland Univ. Technology, 2007.

9. Gauravaram P., Kelsey J. Linear-XOR and additive checksums don't protect Damgaard-Merkle hashes from generic attacks // CT-RSA. Lect. Notes Comput. Sci. - 2008. - V. 4964. - P. 36-51.

10. Joux A. Multicollisions in iterated hash functions. Application to cascaded constructions // CRYPTO’04. Lect. Notes Comput. Sci. - 2004. - V. 3152. — P. 306-316.

11. Kelsey J., Kohno T. Herding hash functions and the Nostradamus attack // EUROCRYPT'06. Lect. Notes Comput. Sci. - 2006. - V. 4004. - P. 183-200.

12. Kelsey J., Schneier B. Second preimages on $n$-bit hash functions for much less than $2^{n}$ work // EUROCRYPT'05. Lect. Notes Comput. Sci. - 2005. V. 3494. - P. 474-490.

13. Lei D. F-HASH: Securing hash functions using Feistel chaining. - 2005. http://eprint.iacr.org/2005/430.pdf

14. Mendel F., Pramstaller N., Rechberger C. A (second) preimage attack on the GOST hash function // FSE'08. Lect. Notes Comput. Sci. - 2008. V. 5086. - P. 224-234. 
15. Mendel F., Pramstaller N., Rechberger C., Kontak M., Szmidt J. Cryptanalysis of the GOST hash function // CRYPTO'08. Lect. Notes Comput. Sci. 2008. - V. 5157. - P. 162-178.

16. Merkle R. C. One way hash functions and DES // CRYPTO'89. Lect. Notes Comput. Sci. - 1990. - V. 435. - P. 428-446.

17. Rivest $R$. The MD5 message-digest algorithm. Request for comments (RFC) 1321, Internet Activities Board, Internet Privacy Task Force, April 1992.

18. Oorschot P.C., Wiener M.J. Parallel collision search with cryptanalytic applications // J. Cryptology. - 1999. - V. 12. № 1. - P. 1-28.

19. Wagner D. A generalized birthday problem // CRYPTO'02. Lect. Notes Comput. Sci. - 2002. - V. 2442. - P. 288-303. 